\title{
Recognizing dualizing complexes
}

\author{
by \\ Peter Jørgensen (København)
}

\begin{abstract}
Let $A$ be a noetherian local commutative ring and let $M$ be a suitable complex of $A$-modules. It is proved that $M$ is a dualizing complex for $A$ if and only if the trivial extension $A \ltimes M$ is a Gorenstein differential graded algebra. As a corollary, $A$ has a dualizing complex if and only if it is a quotient of a Gorenstein local differential graded algebra.
\end{abstract}

Let $A$ be a noetherian local commutative ring and let $M$ be a complex of $A$-modules with homology H $M$ non-zero and finitely generated and $\mathrm{H}_{i} M=0$ for $i<0$. Theorem 2.2 shows that $M$ is a dualizing complex for $A$ if and only if the trivial extension $A \ltimes M$ is a Gorenstein differential graded algebra (DGA). Phrased as a slogan: DGAs can be used to recognize dualizing complexes.

In Corollary 2.3 this is used to show that $A$ has a dualizing complex if and only if it is a quotient of a Gorenstein local DGA.

The notion of Gorenstein DGA I shall use is the one from [5]; it is recalled in Definition 1.3. But note that for the DGAs in Theorem 2.2 and Corollary 2.3, the condition of being Gorenstein can also be expressed by the familar equation $\operatorname{dim}_{\ell} \operatorname{Ext}_{R}(\ell, R)=1$ (see Remark 2.4). DGAs satisfying this equation were considered at length in [1].

A brief introduction to the theory of DGAs is in [5].

1. Definitions. When $A$ is a noetherian commutative ring, $\mathrm{D}(A)$ denotes the derived category of complexes of $A$-modules, and $\mathrm{D}^{\mathrm{f}}(A)$ denotes the full subcategory of complexes $M$ such that $\mathrm{H} M$ is a finitely generated module over $A$. The following definition is due to [6, Def., p. 258].

Definition 1.1. Let $A$ be a noetherian local commutative ring. The complex $D$ in $\mathrm{D}^{\mathrm{f}}(A)$ is called a dualizing complex for $A$ if the canonical

2000 Mathematics Subject Classification: 13D25, 16E45.

Key words and phrases: Noetherian local commutative ring, dualizing complex, trivial extension, Gorenstein differential graded algebra, dualizing differential graded module. 
morphism

$$
A \rightarrow \mathrm{RHom}_{A}(D, D)
$$

is an isomorphism, and $D$ has finite injective dimension.

If a dualizing complex exists, then it is unique up to suspension as follows easily from [6, Thm. V.3.1], but existence is delicate (see [6, Sec. 10]).

Definition 1.2. Let $R$ be a commutative DGA and let $M$ be a differential graded $R$-module (DG $R$-module). Then $R \oplus M$ is again a DG $R$-module with differential

$$
\partial^{R \oplus M}\left(\begin{array}{c}
r \\
m
\end{array}\right)=\left(\begin{array}{c}
\partial^{R} r \\
\partial^{M} m
\end{array}\right)
$$

and the product

$$
\left(\begin{array}{c}
r_{1} \\
m_{1}
\end{array}\right) \cdot\left(\begin{array}{c}
r_{2} \\
m_{2}
\end{array}\right)=\left(\begin{array}{c}
r_{1} r_{2} \\
r_{1} m_{2}+m_{1} r_{2}
\end{array}\right)
$$

turns $R \oplus M$ into a DGA called the trivial extension of $R$ by $M$, denoted by $R \ltimes M$.

Observe that there are canonical morphisms of DGAs,

$$
\begin{aligned}
& R \rightarrow R \ltimes M \rightarrow R, \\
& r \mapsto\left(\begin{array}{c}
r \\
0
\end{array}\right), \\
&\left(\begin{array}{c}
r \\
m
\end{array}\right) \mapsto r,
\end{aligned}
$$

and that the second is a surjection whose kernel is $M$ which can be viewed as a square zero differential graded ideal in $R \ltimes M$.

When $R$ is a commutative DGA with $\mathrm{H}_{0} R$ a noetherian ring, $\mathrm{D}(R)$ denotes the derived category of DG $R$-modules, and $\mathrm{D}^{\mathrm{f}}(R)$ denotes the full subcategory of DG modules $M$ such that H $M$ is a finitely generated module over $\mathrm{H}_{0} R$. (This is compatible with the use of the notation $\mathrm{D}^{\mathrm{f}}$ given before Definition 1.1.) For commutative DGAs, the definitions of Gorenstein DGAs and dualizing DG modules from [5] and [4] simplify as follows.

Definition 1.3. Let $R$ be a commutative DGA with $\mathrm{H}_{0} R$ a noetherian ring. Then $R$ is called Gorenstein if it satisfies:

(i) For $M$ in $\mathrm{D}^{\mathrm{f}}(R)$, the following biduality morphism is an isomorphism:

$$
M \rightarrow \mathrm{RHom}_{R}\left(\mathrm{RHom}_{R}(M, R), R\right) .
$$

(ii) The functor $\mathrm{RHom}_{R}(-, R)$ sends $\mathrm{D}^{\mathrm{f}}(R)$ to itself.

Definition 1.4. Let $R$ be a commutative DGA with $\mathrm{H}_{0} R$ a noetherian ring. The DG $R$-module $E$ is called a dualizing $D G$ module for $R$ if it satisfies: 
(i) The canonical morphism $R \rightarrow \mathrm{RHom}_{R}(E, E)$ is an isomorphism.

(ii) For $M$ in $\mathrm{D}^{\mathrm{f}}(R)$ and for $L$ equal to either $R$ or $E$, the following evaluation morphism is an isomorphism:

$$
M \stackrel{\mathrm{L}}{\otimes}{ }_{R} \operatorname{RHom}_{R}(L, E) \rightarrow \operatorname{RHom}_{R}\left(\operatorname{RHom}_{R}(M, L), E\right) .
$$

(iii) The functor $\mathrm{RHom}_{R}(-, E)$ sends $\mathrm{D}^{\mathrm{f}}(R)$ to itself.

It is clear that $R$ is a Gorenstein DGA if and only if it is a dualizing DG module for itself.

Definition 1.5. Let $R$ be a DGA. Then $R$ is called local if it satisfies:

(i) $R$ is commutative and concentrated in non-negative homological degrees.

(ii) $\mathrm{H}_{0} R$ is a noetherian local ring, and $\mathrm{H} R$ is a finitely generated module over $\mathrm{H}_{0} R$.

(iii) $R_{0}$ is a noetherian ring.

The residue class field $\ell$ of $\mathrm{H}_{0} R$ can then be viewed as a DG $R$-module concentrated in degree 0 , and as such is referred to as the residue class field of $R$.

\section{Results}

Lemma 2.1. Let $A$ be a noetherian local commutative ring, let $R$ be a commutative DGA with $\mathrm{H}_{0} R$ a noetherian ring, and let $R \rightarrow A$ be a morphism of DGAs which induces a surjection $\mathrm{H}_{0} R \rightarrow A$. If $R$ is Gorenstein, then

$$
D=\operatorname{RHom}_{R}(A, R)
$$

is a dualizing complex for $A$.

Proof. Observe that the morphism $R \rightarrow A$ can be used to view any complex of $A$-modules ${ }_{A} M$ as a DG $R$-module ${ }_{R} M$. In other words, any ${ }_{A} M$ in $\mathrm{D}(A)$ can be viewed as ${ }_{R} M$ in $\mathrm{D}(R)$. As $\mathrm{H}_{0} R \rightarrow A$ is surjective, it is clear that

$$
{ }_{A} M \in \mathrm{D}^{\mathrm{f}}(A) \Leftrightarrow{ }_{R} M \in \mathrm{D}^{\mathrm{f}}(R) .
$$

First, (1) implies that $A$ viewed over $R$ is in $\mathrm{D}^{\mathrm{f}}(R)$. Since $R$ is Gorenstein, $D=\mathrm{RHom}_{R}(A, R)$ is then also in $\mathrm{D}^{\mathrm{f}}(R)$, and by (1) this shows that $D$ is in $\mathrm{D}^{\mathrm{f}}(A)$. 
Secondly, there are canonical isomorphisms

$$
\begin{aligned}
\operatorname{RHom}_{A}(D, D) & \stackrel{(\mathrm{a})}{=} \operatorname{RHom}_{A}\left(\operatorname{RHom}_{R}(A, R), \operatorname{RHom}_{R}(A, R)\right) \\
& \stackrel{(\mathrm{b})}{\cong} \operatorname{RHom}_{R}\left(A \stackrel{\mathrm{L}}{A}_{A} \operatorname{RHom}_{R}(A, R), R\right) \\
& \cong \operatorname{RHom}_{R}\left(\operatorname{RHom}_{R}(A, R), R\right) \\
& \stackrel{(\mathrm{c})}{\cong} A
\end{aligned}
$$

where (a) is by the definition of $D$ and (b) is by adjointness, while (c) is because $R$ is Gorenstein.

Thirdly, let $k$ be the residue class field of $A$. There are isomorphisms

$$
\begin{aligned}
\operatorname{RHom}_{A}(k, D) & \stackrel{(\mathrm{d})}{=} \operatorname{RHom}_{A}\left(k, \operatorname{RHom}_{R}(A, R)\right) \\
& \stackrel{(\mathrm{e})}{\cong} \operatorname{RHom}_{R}(A \stackrel{\mathrm{L}}{\otimes} k, R) \\
& \cong \operatorname{RHom}_{R}(k, R),
\end{aligned}
$$

where again (d) is by the definition of $D$ and (e) is by adjointness. (1) implies that $k$ viewed over $R$ is in $\mathrm{D}^{\mathrm{f}}(R)$, and since $R$ is Gorenstein, $\operatorname{RHom}_{R}(k, R)$ is then also in $\mathrm{D}^{\mathrm{f}}(R)$ so has bounded homology. By (2), the same holds for $\operatorname{RHom}_{A}(k, D)$, and then by [2, (A.5.7.4)] the injective dimension $\operatorname{id}_{A} D$ is finite.

Altogether, $D$ is a dualizing complex for $A$ (cf. Definition 1.1).

THEOREM 2.2. Let $A$ be a noetherian local commutative ring and let $M$ in $\mathrm{D}^{\mathrm{f}}(A)$ have $M \neq 0$ and $\mathrm{H}_{i} M=0$ for $i<0$. Then

$M$ is a dualizing complex for $A \Leftrightarrow A \ltimes M$ is a Gorenstein $D G A$.

Proof. $\Rightarrow$ : Suppose that $M$ is a dualizing complex for $A$. Clearly, $A \ltimes M$ can be viewed as a DGA over $A$ with $\mathrm{H}(A \ltimes M)$ finitely generated over $A$. Hence [4, Prop. 2.6] says that $A \ltimes M$ has the dualizing DG module $\operatorname{RHom}_{A}(A \ltimes M, M)$.

Let

$$
M \stackrel{\varrho}{\rightarrow} I
$$

be an injective resolution. Then $\operatorname{RHom}_{A}(A \ltimes M, M)$ is isomorphic to

$$
E=\operatorname{Hom}_{A}(A \ltimes M, I),
$$

so $E$ is a dualizing DG module for $A \ltimes M$. The $(A \ltimes M)$-structure of $E$ comes from the $A \ltimes M$ appearing in the first variable of the Hom; that is, if $\varepsilon$ is a graded element of $E$ and $r_{1}$ and $r_{2}$ are graded elements of $A \ltimes M$, then

$$
\left(r_{1} \varepsilon\right)\left(r_{2}\right)=(-1)^{\left|r_{1}\right|\left(|\varepsilon|+\left|r_{2}\right|\right)} \varepsilon\left(r_{2} r_{1}\right)
$$


Now note that

$$
E=\operatorname{Hom}_{A}(A \ltimes M, I) \cong \operatorname{Hom}_{A}(A, I) \oplus \operatorname{Hom}_{A}(M, I)
$$

as complexes of $A$-modules. This enables me to compute henceforth as if the elements of $E$ were column vectors $\left(\begin{array}{l}\alpha \\ \mu\end{array}\right)$ where $A \stackrel{\alpha}{\rightarrow} I$ and $M \stackrel{\mu}{\rightarrow} I$ are $A$-linear. Of course, I must remember that $E$ is a DG $(A \ltimes M)$-module via $(3)$.

The element $\left(\begin{array}{l}0 \\ \varrho\end{array}\right)$ in $E$ can be used to define a morphism of DG $(A \ltimes M)$ modules by

$$
A \ltimes M \stackrel{\varphi}{\rightarrow} E, \quad \varphi\left(\left(\begin{array}{c}
a \\
m
\end{array}\right)\right)=\left(\begin{array}{c}
a \\
m
\end{array}\right) \cdot\left(\begin{array}{l}
0 \\
\varrho
\end{array}\right) .
$$

In fact, this turns out to be an isomorphism when viewed in $\mathrm{D}(A \ltimes M)$. Hence $A \ltimes M$ is a dualizing DG module for itself, and so $A \ltimes M$ is Gorenstein as desired.

To see this, one uses $(3)$ to get the second $=$ in

$$
\varphi\left(\left(\begin{array}{l}
a \\
m
\end{array}\right)\right)=\left(\begin{array}{l}
a \\
m
\end{array}\right) \cdot\left(\begin{array}{l}
0 \\
\varrho
\end{array}\right)=\left(\begin{array}{c}
\chi \varrho(m) \\
a \varrho
\end{array}\right),
$$

where $\chi_{i}$ denotes the morphism $A \rightarrow I$ defined by $\chi_{i}(a)=a i$ for any $i$ in $I$. This shows that viewed in $\mathrm{D}(A)$, the morphism $\varphi$ is the obvious morphism which identifies $A \oplus M$ with

$$
\begin{aligned}
E & \cong \operatorname{Hom}_{A}(A, I) \oplus \operatorname{Hom}_{A}(M, I) \\
& \cong \operatorname{RHom}_{A}(A, M) \oplus \operatorname{RHom}_{A}(M, M) \\
& \cong M \oplus A .
\end{aligned}
$$

So $\varphi$ is an isomorphism when viewed in $\mathrm{D}(A)$. Hence $\mathrm{H} \varphi$ is bijective, and so $\varphi$ is also an isomorphism when viewed in $\mathrm{D}(A \ltimes M)$.

$\Leftarrow$ : Suppose that $A \ltimes M$ is a Gorenstein DGA. Replace $M$ with a quasiisomorphic complex which consists of finitely generated modules and satisfies $M_{i}=0$ for $i<0$. Thereby $A \ltimes M$ is replaced with a quasi-isomorphic DGA which is local. It is clear that the new $A \ltimes M$ remains Gorenstein, and that it is enough to show that the new $M$ is a dualizing complex for $A$.

By the remarks after Definition 1.2, there is a canonical morphism $A \ltimes M$ $\rightarrow A$ of DGAs, and it is clear that this induces a surjection $\mathrm{H}_{0}(A \ltimes M) \rightarrow A$. So since $A \ltimes M$ is a Gorenstein DGA, Lemma 2.1 shows that $A$ has a dualizing complex $D$.

Now, [4, Prop. 2.6] implies that $A \ltimes M$ has the dualizing DG module $\mathrm{RHom}_{A}(A \ltimes M, D)$. On the other hand, $A \ltimes M$ is Gorenstein, so it is a dualizing DG module for itself. By [4, Thm. 3.2] this implies that $\mathrm{RHom}_{A}(A \ltimes M, D)$ and $A \ltimes M$ are isomorphic up to suspension in $\mathrm{D}(A \ltimes M)$. 
Replacing $D$ with a (positive or negative) suspension, I can therefore suppose that there is an isomorphism in $\mathrm{D}(A \ltimes M)$,

$$
A \ltimes M \stackrel{\cong}{\rightrightarrows} \operatorname{RHom}_{A}(A \ltimes M, D) .
$$

Picking an injective resolution $D \rightarrow I$ gives that $\operatorname{RHom}_{A}(A \ltimes M, D)$ is isomorphic to

$$
E=\operatorname{Hom}_{A}(A \ltimes M, I),
$$

so all in all there is an isomorphism in $\mathrm{D}(A \ltimes M)$,

$$
A \ltimes M \stackrel{\cong}{\rightrightarrows} E .
$$

This can be represented by two quasi-isomorphisms of DG $(A \ltimes M)$-modules,

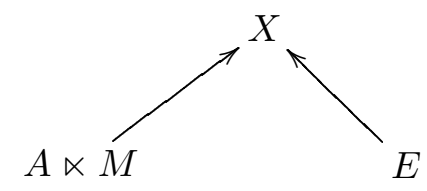

and as $A \ltimes M$ is a free DG module over itself, the left-hand arrow lifts through the right-hand arrow modulo homotopy, and hence gives a quasiisomorphism of DG $(A \ltimes M)$-modules,

$$
A \ltimes M \stackrel{\varphi}{\rightarrow} E .
$$

Now use again the decomposition of $E$ given in (4), and suppose

$$
\varphi\left(\left(\begin{array}{l}
1 \\
0
\end{array}\right)\right)=\left(\begin{array}{l}
\alpha \\
\mu
\end{array}\right)
$$

One can then use (3) to get the last $=$ in

$$
\varphi\left(\left(\begin{array}{c}
a \\
m
\end{array}\right)\right)=\varphi\left(\left(\begin{array}{c}
a \\
m
\end{array}\right) \cdot\left(\begin{array}{l}
1 \\
0
\end{array}\right)\right)=\left(\begin{array}{c}
a \\
m
\end{array}\right) \cdot \varphi\left(\left(\begin{array}{l}
1 \\
0
\end{array}\right)\right)=\left(\begin{array}{c}
\chi_{\alpha(a)+\mu(m)} \\
a \mu
\end{array}\right),
$$

where again $\chi_{i}$ denotes the morphism $A \rightarrow I$ defined by $\chi_{i}(a)=a i$ for any $i$ in $I$. This means that if $\varphi$ is viewed as a morphism of complexes of $A$-modules,

$$
A \oplus M \stackrel{\varphi}{\rightarrow} \operatorname{Hom}_{A}(A, I) \oplus \operatorname{Hom}_{A}(M, I),
$$

then $\varphi$ is given by the matrix

$$
\varphi=\left(\begin{array}{cc}
\chi_{\alpha(-)} & \chi_{\mu(-)} \\
\mu \cdot & 0
\end{array}\right)
$$

The triangular form of the matrix implies that there is a commutative dia- 
gram of complexes of $A$-modules,

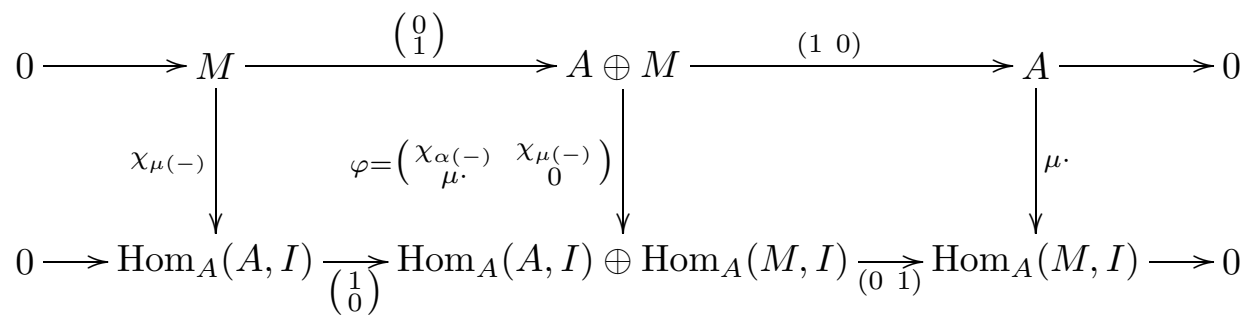

which can be written more simply as

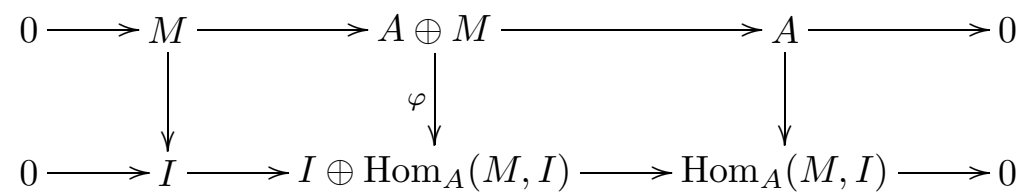

Applying to this the functor $\operatorname{Hom}_{A}(P,-)$, where $P \rightarrow k$ is a projective resolution of $k$, the residue class field of $A$, yields a new commutative diagram of complexes of $A$-modules, with split exact rows (because the above diagram has split exact rows), and with the middle vertical morphism a quasi-isomorphism (because the same holds for $\varphi$ in the above diagram, and because $\operatorname{Hom}_{A}(P,-)$ preserves quasi-isomorphisms).

The new commutative diagram of complexes of $A$-modules induces a commutative diagram of long exact sequences of homology groups. Since $P$ is a projective resolution of $k$, the homology groups are certain $\operatorname{Ext}_{A}^{i}(k,-)$ 's. Moreover, the connecting homomorphisms are zero (because the rows in the diagram of complexes are split exact), and the vertical morphisms which result from the middle vertical morphism in the diagram of complexes are isomorphisms (because the middle vertical morphism in the diagram of complexes is a quasi-isomorphism).

Summing up, this gives for each $i$ a commutative diagram with exact rows,

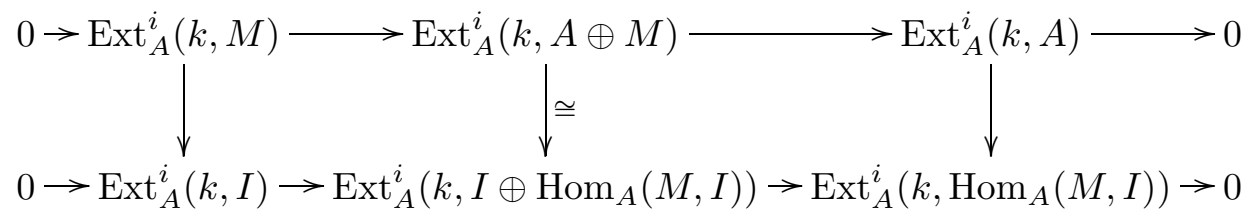

In particular, $\operatorname{Ext}_{A}^{i}(k, M) \rightarrow \operatorname{Ext}_{A}^{i}(k, I)$ is injective for each $i$, so

$$
\operatorname{dim}_{k} \operatorname{Ext}_{A}^{i}(k, M) \leq \operatorname{dim}_{k} \operatorname{Ext}_{A}^{i}(k, I)=\operatorname{dim}_{k} \operatorname{Ext}_{A}^{i}(k, D)
$$

for each $i$. But $\operatorname{dim}_{k} \operatorname{Ext}_{A}^{i}(k, D)=\delta_{i e}$ for some fixed $e$ by [6, Prop. V.3.4], where $\delta_{i e}$ is 1 for $i=e$ and 0 otherwise. So the only possibilities for $\operatorname{dim}_{k} \operatorname{Ext}_{A}^{i}(k, M)$ are $\delta_{i e}$ or identically zero. 
The latter alternative would give $\operatorname{depth}_{A} M=\infty$, which is impossible by [2, Lemma (A.8.9)] because of $M \neq 0$. So the former alternative

$$
\operatorname{dim}_{k} \operatorname{Ext}_{A}^{i}(k, M)=\delta_{i e}
$$

must hold, and then $M$ is a dualizing complex for $A$ by [6, Prop. V.3.4] again.

Corollary 2.3. Let $A$ be a noetherian local commutative ring. Then $A$ has a dualizing complex if and only if it is a quotient of a Gorenstein local $D G A$.

Proof. Suppose that $A$ is a quotient of $R$ which is a Gorenstein local DGA, and let $R \rightarrow A$ be the quotient morphism. As $R$ is concentrated in non-negative degrees, this clearly induces a surjection $\mathrm{H}_{0} R \rightarrow A$. So $D=\mathrm{RHom}_{R}(A, R)$ is a dualizing complex for $A$ by Lemma 2.1.

On the other hand, suppose that $A$ has a dualizing complex $D$. By replacing $D$ with a high suspension, I can suppose $\mathrm{H}_{i} D=0$ for $i<0$, and then by replacing $D$ with a quasi-isomorphic complex, that $D$ consists of finitely generated modules and satisfies $D_{i}=0$ for $i<0$. Then $A \ltimes D$ is a local DGA, and as $D$ is a dualizing complex for $A$, Theorem 2.2 shows that $A \ltimes D$ is a Gorenstein DGA. And $A \ltimes D$ has $A$ as a quotient by the remark after Definition 1.2.

Remark 2.4. Theorem 2.2 and Corollary 2.3 concern DGAs which are Gorenstein in the sense of Definition 1.3. However, for the DGAs in question, this condition can be expressed in an alternative, simple way:

In $[4$, Thm. 4.3$]$ it was proved that if $R$ is a local DGA with residue class field $\ell$, then

$$
R \text { is Gorenstein } \Leftrightarrow \operatorname{dim}_{\ell} \operatorname{Ext}_{R}(\ell, R)=1 \text {. }
$$

Corollary 2.3 deals with a local DGA to which (6) applies.

Theorem 2.2 does not deal directly with a local DGA. However, I can replace $M$ in Theorem 2.2 with a quasi-isomorphic complex which consists of finitely generated modules and satisfies $M_{i}=0$ for $i<0$. Thereby $A \ltimes M$ is replaced with a quasi-isomorphic DGA which is local and to which (6) applies. And it is clear that the old and new $A \ltimes M$ are Gorenstein simultaneously.

Acknowledgements. Precursors to the above results have been mentioned in conversations I have had with Amnon Yekutieli and Anders Frankild.

The principle of recognizing dualizing complexes by means of trivial extensions is well known in the Cohen-Macaulay case (see [3, Prop. 4.2] and [9, Thm. (7)]). 
By [8, Thm. 1.2], a stronger result than Corollary 2.3 holds, namely, $A$ has a dualizing complex if and only if it is a quotient of a Gorenstein noetherian local commutative ring $R$.

A different way of using DGAs to recognize dualizing complexes is in [7].

\section{References}

[1] L. L. Avramov and H.-B. Foxby, Locally Gorenstein homomorphisms, Amer. J. Math. 114 (1992), 1007-1047.

[2] L. W. Christensen, Gorenstein Dimensions, Lecture Notes in Math. 1747, Springer, Berlin, 2000.

[3] H.-B. Foxby, Gorenstein modules and related modules, Math. Scand. 31 (1972), 267284 .

[4] A. Frankild, S. Iyengar and P. Jørgensen, Dualizing DG modules and Gorenstein DG algebras, J. London Math. Soc., to appear.

[5] A. Frankild and P. Jørgensen, Gorenstein Differential Graded Algebras, Israel J. Math., in press.

[6] R. Hartshorne, Residues and Duality, Lecture Notes in Math. 20, Springer, Berlin, 1966. Notes of a seminar on the work of A. Grothendieck, given at Harvard 1963/64. With an appendix by P. Deligne.

[7] V. Hinich, Rings with approximation property admit a dualizing complex, Math. Nachr. 163 (1993), 289-296.

[8] T. Kawasaki, On Macaulayfication of noetherian schemes, Trans. Amer. Math. Soc. 352 (2000), 2517-2552.

[9] I. Reiten, The converse to a theorem of Sharp on Gorenstein modules, Proc. Amer. Math. Soc. 32 (1972), 417-420.

Danish National Library of Science and Medicine

Nørre Allé 49

2200 København N, DK-Denmark

E-mail: pej@dnlb.dk

Web: www.geocities.com/popjoerg 